\title{
Entropy, Nonequilibrium, Chaos and Infinitesimals
}

\author{
Giovanni Gallavotti ${ }^{1}$ \\ ${ }^{1}$ Dipartimento di Fisica and INFN, Università di Roma La Sapienza, P. A. Moro 2, 00185, Roma, Italy
}

(Dated: January 2, 2018)

A survey of the approach to Statistical Mechanics following Boltzmann's theory of ensembles and ergodic hypothesis leading to chaoticity as a unifying principle of equilibrium and nonequilibrium Statistical Mechanics.

PACS numbers: 47.52.+j, 05.45.-a, 05.70.Ln, 05.20.-y

\section{BOLTZMANN AND ENTROPY}

Since the earliest works BoLTZMANN aimed at a microscopic interpretation or "proof" of the newly formulated second Law of Thermodynamics and of the associated concept of entropy, [1]. At the time, since the works of Bernoulli, Avogadro, Herapath, Waterstone, KRÖNIG, Clausius it was well established that there should be an identification between absolute temperature and average kinetic energy at least in gas theory.

BoLTZMANN starts in [1] by stating clearly that in general (i.e. not only for gases) temperature and average kinetic energy must be identified and gives a derivation of the second law for isocore (i.e. constant volume) transformations. This first derivation makes use of a periodicity assumption on the motion of (each) gas particle to obtain the existence of the time average of the kinetic energy and seems to fail if the motions are not periodic.

Nevertheless Boltzmann insisted in conceiving aperiodic motions as periodic with infinite period: see [1] where on p. 30 one finds that "... this explanation is nothing else but the mathematical formulation of the theorem according to which the paths that do not close themselves in any finite time can be regarded as closed in an infinite time". He therefore pursued the implied mechanical proof of Thermodynamics to the extreme consequences.

A "proof" of the second law meant to look for properties of the trajectories in phase space of a mechanical equation of motion, like time averages of suitable observables, which could have the interpretation of thermodynamic quantities like pressure $p$, volume $V$, energy $U$, temperature $T$, entropy $S$ and be related by the thermodynamic relations, namely

$$
d S=\frac{d U+p d V}{T}
$$

where $d U, d V, d S$ are the variations of $U, V, S$ when the control parameters of the system are infinitesimally changed.

The extreme consequence was the ergodic hypothesis which was first mentioned by BolTzMANN around 1870, see p. 237 in [2], for the internal motion of atoms in single molecules of a gas ("it is clear that the various gas molecules will go through all possible states of motion" which, however, could possibly be understood from the context to be different from the ergodic hypothesis, see [3], because the molecules undergo from time to time collisions). See also p. 96 in [4] and p. xxxvii in the recent collection by FLAMM [5].

Considering a collection of copies of the system alike to a large molecule, p. 284 in [6], the same assumption became what is often referred as the ergodicity property of the entire gas. It implied that, by considering all motions periodic, kinetic energy equipartition would follow and, better (see p. 287 in [6]), even what we call now the microcanonical distribution would follow (as well as the canonical distribution). The hypothesis was taken up also by MAXWELL (1879), see p. 506 in [3]).[53]

In this way BoLtzmanN was able to derive various thermodynamic consequences and a proof of 1 , see [7], and was led to exhibiting a remarkable example of what later would be called a "thermodynamic analogy" (Sec.III of [7]). This meant the existence of quantities associated with the phase space of a mechanical equation of motion (typically defined as time averages over the solutions of the equations of motion), which could be given thermodynamic names like equilibrium state, pressure $p$, volume $V$, energy $U$, temperature $T$, entropy $S$ and be related by the thermodynamic relations that are expected to hold between the physical quantities bearing the same name, namely 1.

The notion of mechanical thermodynamic analogy was formulated and introduced by HeLMoLTz for general systems admitting only periodic motions (called monocyclic), $[8,9]$. The proposal provided a new perspective and generated the new guiding idea that the Thermodynamic relations would hold in every mechanical system, from the small and simple to the large and complex: in the first cases the relations would be trivial identities of no or little interest, just thermodynamic analogies, but in the large systems they would become nontrivial and interesting being relations of general validity. In other words they would be a kind of symmetry property of Hamiltonian Mechanics.

The case of spatially confined systems with one degree of freedom was easy (easier than the example already 
given in [7]): with all motions periodic, the microscopic state was indentified with the phase space point $(p, q)$ representing the full mechanical state of the system, the macroscopic state in the corresponding thermodynamic analogy was identified with the energy surface $H(p, q)=$ $\frac{1}{2 m} p^{2}+\varphi_{V}(q)=U$, where $m$ is the mass and $\varphi_{V}$ is the potential energy which confines the motion in position space, i.e. in $q$, and depends on a parameter $V$. The state is completely determined by two parameters $U, V$.

Average kinetic energy $T=\lim _{\tau \rightarrow \infty} \frac{1}{\tau} \int_{0}^{\tau} K(p(t)) d t$ is identified with temperature; energy is identified with $U$ : then if pressure $p$ is defined as the time average $\lim _{\tau \rightarrow \infty}-\frac{1}{\tau} \int_{0}^{\tau} \partial_{V} \varphi_{V}(q(t)) d t$ the quantities $T, p$ become functions $p=p(U, V), T=T(U, V)$ of the parameters $U, V$ determining the state of the system and the 1 should hold.

Indeed the limits as $\tau \rightarrow \infty$ exist in such a simple case, in which all motions are periodic and confined between $q_{ \pm}=q_{ \pm}(U, V)$ (where $U=\varphi_{V}\left(q_{ \pm}\right)$); it is $d t \equiv \frac{d q}{|\dot{q}|}=$ $\frac{d q}{\sqrt{2\left(U-\varphi_{V}(q)\right) / m}}$ and the period of the oscillations is $\tau_{0}=$ $\tau_{0}(U, V)=2 \int_{q_{-}}^{q_{+}} \frac{d q}{\sqrt{2\left(U-\varphi_{V}(q)\right) / m}}$, hence (p. 127 in [10] and Ch. I in [11]),

$$
\begin{array}{r}
T=\frac{2}{\tau_{0}} \int_{q_{-}(U, V)}^{q_{+}(U, V)} \frac{m}{2} \sqrt{\frac{2}{m}\left(U-\varphi_{V}(q)\right)} d q, \\
p=\frac{2}{\tau_{0}} \int_{q_{-}(U, V)}^{q_{+}(U, V)} \frac{\partial_{V} \varphi_{V}(q)}{\sqrt{\frac{m}{2}\left(U-\varphi_{V}(q)\right)}} d q
\end{array}
$$

and it is immediate to check, as in [7], that 1 is fulfilled by setting

$$
\begin{gathered}
S(U, V)=2 \log \int_{H=U} p d q= \\
=2 \log \int_{q_{-}(U, V)}^{q_{+}(U, V)} \sqrt{2 m\left(U-\varphi_{V}(q)\right)} d q
\end{gathered}
$$

The case of the central motion studied in [7] was another instance of monocyclic systems, i.e. systems with only periodic motions.

Then in the fundamental paper [10], following and inspired by the quoted works of HeLmoltz, Boltzmann was able to achieve what I would call the completion of his program of deducing the second law (1) from Mechanics. If

(1) the absolute temperature $T$ is identified with the average kinetic energy over the periodic motion following the initial datum $(\mathbf{p}, \mathbf{q})$ of a macroscopic collection of $N$ identical particles interacting with a quite arbitrary pair interaction, and

(2) the energy $U$ is $H(\mathbf{p}, \mathbf{q})$ sum of kinetic and of a potential energy,

(3) the volume $V$ is the volume of the region where the positions $\mathbf{q}$ are confined (typically by a hard wall potential),

(4) the pressure $p$ is the average force exercized on the walls by the colliding particles,

then, from the assumption that each point would evolve periodically visiting every other point on the energy surface (i.e. assuming that the system could be regarded as monocyclic, see [11] Appendix 9.3 for details) it would follow that the quantity $p$ could be identified with the $\left\langle-\partial_{V} \varphi_{V}\right\rangle$, time average of $-\partial_{V} \varphi_{V}$, and 1 would follow as a heat theorem. The heat theorem would therefore be a consequence of the general properties of monocyclic systems.

This led Boltzmann to realize, in the same paper, that there were a large number of mechanical models of Thermodynamics: the macroscopic states could be identified with regions of phase spaces invariant under time evolution and their points would contribute to the average values of the quantities with thermodynamic interpretation (i.e. $p, V, U, T$ ) with a weight (hence a probability) also invariant under time evolution.

Hence imagining the weights as a density function one would see the evolution as a motion of phase space points leaving the density fixed. Such distributions on phase space were called monodic (because they keep their identity with time or, as we say, are invariant): and in [10] several collections of weights or monodes were introduced: today we call them collections of invariant distributions on phase space or ensembles. Among the ensembles $\mathcal{E}$, i.e. collections of monodes, BoltzMANN singled out the ensembles called orthodes ("behaving correctly"): they were the families of probability distributions depending on a few parameters (normally 2 for simple one component systems) such that the corresponding averages $p, V, U, T$, defined in (1-4) above, would vary when the parameters were varied causing variations $d U, d V$ of average energy and volume in such a way that the r.h.s. of 1 would be an exact differential, thereby defining the entropy $S$ as a function of state, see [11, 12].

The ergodic hypothesis yields the "orthodicity" of the ensemble $\mathcal{E}$ that today we call microcanonic (in [10] it was named ergode): but ergodicity, i.e. the dynamical property that evolution would make every phase space point visit every other, was not necessary to the orthodicity proof of the ergode. In fact in [10] the relation 1 is proved directly without recourse to dynamical properties (as we do today, see $[11,13,14]$ ); and in the same way the orthodicity of the canonical ensemble (called holode in [10]) was obtained and shown to generate a Thermodynamics which is equivalent to the one associated with the microcanonical ensemble. [54]

In the end in [6] and, in final form, in [10] the theory of ensembles and of their equivalence was born without need of the ergodic property: the still important role of the ergodic hypothesis was to guarantee that the quantities $p, V, U, T, S$ defined by orthodic averages with respect to invariant distributions on phase space had the physical meaning implied by their names (this was true for the microcanonical ensemble by the ergodic hypothesis, and for the other ensembles by the equivalence).

At the same time entropy had received a full microscopic interpretation consistent with, but independent of, the one arising from the Boltzmann's equation in the rar- 
efied gases case, which can be seen as a quite independent development of Boltzmann's work. Furthermore it became clear that the entropy could be identified, up to a universal proportionality constant $k_{B}$, with the volume of phase space enclosed by the energy surface.

Unfortunately the paper [10] has been overlooked until quite recently by many, actually by most, physicists possibly because it starts, in discussing the thermodynamic analogy, by giving the Saturn rings as an "example": a brilliant one, certainly but perhaps discouraging for the suspicious readers of this deep and original paper on Thermodynamics. See p.242 and p. 368 in [15] for an exception, possibly the first.

\section{BOLTZMANN'S DISCRETE VISION OF THE ERGODIC PROBLEM}

The ergodic hypothesis could not possibly say that every point of the energy surface in phase space visits in due time (the recurrence time) every other, see also p. 505 and following in [3]. But this statement was attributed to Boltzmann and criticized over and over again (even by Physicists, including in the influential book, [16], although enlightened mathematicians could see better, see p.385 in [15]): however for BoLTZMANN phase space was discrete and points in phase space were cells $\Delta$ with finite size, that I will call $h$. And time evolution was a permutation of the cells: ergodicity meant therefore that the permutation was a one cycle permutation.

This conception, perfectly meaningful mathematically, was apparently completely misunderstood by his critics: yet it was clearly stated in one of the replies to ZERMELO, [17], and in the book on gases, [18], see also [12] and the DE Courtenay's communication in this Symposium.

In order to explain how a reversible dynamics could be compatible with the irreversibility of macroscopic phenomena he had, in fact, to estimate the recurrence time. This was done by multiplying the typical time over which a microscopic event (i.e. a collision) generates a variation of the coordinates of an order of magnitude appreciable on microscopic scales (i.e. a time interval of $\sim 10^{-12} \mathrm{~s}$ and a coordinate variation of the order of $1^{\circ} \mathrm{A}$ ) times the number of cells into which phase space was imagined to be subdivided.

The latter number was obtained by dividing the phase space around the energy surface into equal boxes of a size $h$ equal to the $3 N$-th power of $\rho^{-\frac{1}{3}}$ times $\sqrt{m k_{B} T}$ with $\rho$ the numerical density and $k_{B}$ Boltzmann's constant and $T$ temperature. With the data for $\mathrm{H}_{2}$ at normal conditions in $1 \mathrm{~cm}^{3}$ an ealier estimate of Thomson, [19], was rederived (and a recurrence time scale so large that it would be immaterial to measure it in seconds or in ages of the Universe).

Of course conceiving phase space as discrete is essential to formulate the ergodicity property in an acceptable way: it does not, however, make it easier to prove it even in the discrete sense just mentioned (nor in the sense acquired later when it was formulated mathematically for systems with continuous phase space). It is in fact very difficult to be a priori sure that the dynamics is an evolution which has only one cycle. Actually this is very doubtful: as one realizes if one attempts a numerical simulation of an equation of motion which generates motions which are ergodic in the mathematical sense.

And the difficulty is already manifest in the simpler problem of simulating differential equations in a way which rigorously respects the uniqueness theorem. In computers the microscopic states are rigorously realized as cells (because points are described by integers, so that the cells sizes are limited by the precision of hardware and software) and phase space is finite. By construction simulation programs map a cell into another: but it is extremely difficult, and possible only in very special cases (among which the only nontrivial that I know is [20]) without dedicating an inordinate computing time to insure a $1-1$ correspondence between the cells.

Nevertheless the idea that phase space is discrete and motion is a permutation of its points is very appealing because it gives a privileged role to the uniform distribution on the phase space region in which the motion develops (i.e. the energy surface, if the ergodic hypothesis holds). However it is necessary, for consistency, that the phase space cells volume does not change with time, see Ch. 1 in [11]: this is a property that holds for Hamiltonian evolutions and therefore allows us to imagine the ergodic hypothesis as consistent with the predictions of Statistical Mechanics.

\section{BOLTZMANN'S HERITAGE}

The success of the ergodic hypothesis has several aspects. One that will not be considered further is that it is not necessary: this is quite clear as in the end we want to find the relations between a very limited number of observables and we do not need for that an assumption which tells us the values of all possible averages, most of which concern "wild" observables (like the position of a tagged particle). The consequence is that the ergodic hypothesis is intended in the sense that confined Hamiltonian systems "can be regarded as ergodic for the purpose of studying their equilibrium properties".

What is, perhaps, the most interesting aspect of the hypothesis is that it can hold for systems of any size and lead to relations which are essentially size independent as well as model independent and which become interesting properties when considered for macroscopic systems.

Is it possible to follow the same path in studying nonequilibrium phenomena? The simplest such phenomena arise in stationary states of systems subject to the action of nonconservative forces and of suitable heat removing forces (whose function is to forbid indefinite build up of energy in the system).

Such states are realized in Physics with great accuracy 
for very long times, in most cases longer than the available observation times. For instance it is possible to keep a current circulating in a wire subject to an electromotive force for a very long time, provided a suitable cooling device is attached to the wire.

As in equilibrium, the stationary states of a system will be described by a collection of probability distributions on phase space $\mathcal{E}$, invariant with respect to the dynamics, which I call ensemble: the distributions $\mu$ in $\mathcal{E}$ will be parameterized by a few parameters $U, V, E_{1}, E_{2}, \ldots$ which have a physical interpretation of (say) average energy, volume, intensity of the nonconservative forces acting on the system (that will be called "external parameters"). Each distribution $\mu$ will describe a macroscopic state in which the averages of the observables will be their integrals with respect to $\mu$. The equations of motion will be symbolically written as

$$
\dot{\mathbf{x}}=\mathbf{f}(\mathbf{x})
$$

and we shall assume that $\mathbf{f}$ is smooth, that it depends on the external parameters and that the phase space visited by trajectories is bounded (at fixed external parameters and initial data).

Since we imagine that the system is subject to nonconservative forces the phase space volume (or any measure with density with respect to the volume) will not be preserved by the evolution and the divergence

$$
\sigma(\mathbf{x})=-\sum_{i} \partial_{x_{i}} f_{i}(\mathbf{x})
$$

will be different from 0 .

We expect that, in interesting cases, the time average $\sigma_{+}$of $\sigma$ will be positive:

$$
\sigma_{+} \stackrel{\text { def }}{=} \lim _{\tau \rightarrow \infty} \frac{1}{\tau} \int_{0}^{\tau} \sigma\left(S_{t} x\right) d t>0 .
$$

and, with few exceptions, $x$-independent.

This means that there cannnot be invariant distributions with density with respect to the volume. And the problem to find even a single invariant distribution is notrivial, except possibly for the ones concentrated on periodic orbits.

The problem can be attacked, possibly, by following BoltzMAnN's view of dynamics as discrete, ("die Zahl der lebendigen Kräft ist eine diskrete", see p. 167 in [21]).

\section{EXTENDING BOLTZMANN'S ERGODIC HYPOTHESIS.}

Consider a generic "chaotic" system described by equations like 4 which generate motions confined in phase space. Under very general conditions it follows that $\sigma_{+} \geq 0,[22]$, and we concentrate on the case $\sigma_{+}>0$. The suggestion that phase space should be regarded as discrete, and motion should simply be a one-cycle permutation of the "cells" $\Delta$ representing the phase space points is still very appealing as it would lead to the unambiguous determination of the invariant distribution $\mu$ describing the statistical properties of the stationary states.

In fact this is an assumption implicit in any claim of physical relevance of a simulation: as already mentioned above, a computer program defines a map on small cells in phase space. Already in the case of Hamiltonian systems (i.e. in equilibrium theory) a simulation will not respect the uniqueness of solutions of the equation of motion because the map between the cells will not be invertible: it is extremely hard to write a program which avoids that two distinct cells are mapped into the same cell (see above).

When $\sigma_{+}>0$ so that, in the average, phase space volume contracts the uniqueness problem becomes essentially unsurmountable (and not only in simulations); and there will be very many cells that eventually evolve into the same cell: thus the evolution will not be a permutation of the cells. It will, however, become eventually a permutation of a subset of the initial set of cells. This reflects the fact that the orbits of the solutions of the differential equation 4 will "cluster" on an attractor which is a set of 0 volume.

The conclusion is that the statistics of the motions will still be a well defined probability distribution on phase space provided the ergodic hypothesis is extended to mean that the permutation of the cells on the attractor is a one-cycle permutation: it will be, in this case, still the uniform distribution concentrated on the cells lying on the attractor.

This viewpoint unifies the conception of the statistics of equilibrium and of stationary nonequilibrium: the statistics $\mu$ of the motions, i.e. the probability distribution $\mu$ such that, in the continuous version of the models,

$$
\lim _{\tau \rightarrow \infty} \frac{1}{\tau} \int_{0}^{\tau} F\left(S_{t} x\right) d t=\int F(y) \mu(d y)
$$

for all smooth observables $F$ and for all but a set of zero volume of points $x$ on phase space, can be considered, in equilibrium as well as in stationary non equilibrium states, as a probability distribution which is uniform on the attractor.

The key obstacle to the above conception of Statistical Mechanics for stationary states is that phase space cells cannot be supposed to evolve, under the evolution assigned by 4 when $\sigma_{+}>0$, keeping a constant volume. Therefore regarding evolution as a map between cells of a discretized version of phase space contains new sources of possible errors. Besides the error that is present in equilibrium theory due to the cells deformations which leads to violations of the uniqueness, [11], there is an error due to their contraction $\sigma_{+}>0$. 
In equilibrium the first error can be reduced by reducing the cells size and the time intervals at which the observations (to be interpolated into the estimate of the integral in 7) are taken. This is a nontrivial source of errors that can be estimated to be physically acceptable, at least for the evaluation of the averages of the few observables relevant for Thermodynamics, only in certain regions of the phase diagrams, see Ch. I in [11]. But at least in such regions the discrete interpretation of the ergodic hypothesis leads us to a consistent representation of the evolution as a permutation between discrete elements of a partition of phase space into small cells.

Out of equilibrium the further source of discretization error due to the actual reduction of phase space volume implies that it is not consistent to view the motion as a permutation of cells of a discretization of phase space into small equal volume elements.

A possible way out is to restrict attention to systems that show strongly chaotic behavior. For instance systems which are transitive (i.e. admit a dense orbit) and hyperbolic, see [23] for a formal definition, are typically chaotic systems which are also quite well understood.

To enter into some detail it is convenient to look at the time evolution by drawing a few surfaces $\Sigma_{1}, \Sigma_{2}, \ldots, \Sigma_{s}$ transversal to the phase space trajectories, and such that the trajectories cross some of the surfaces over and over again (i.e. each trajectory crosses the surfaces infinitely many times both in the future and in the past). Let $\Sigma=\cup_{j} \Sigma_{j}$ (usually called a "Poincaré's section") and let $S$ be the map which transforms a point $\xi \in \Sigma$ (i.e. on one of the surface elements $\Sigma_{1}, \Sigma_{2}, \ldots, \Sigma_{s}$ ) into the point $S \xi$ where the orbit of $\xi$ meets again for the first time $\Sigma$ (i.e. it is again on one of the surface elements defining $\Sigma)$.

The points in phase space can therefore be described by pairs $x=(\xi, \theta)$ if $\xi$ is the point in $\Sigma$ last visited by the trajectory starting at $x$ and $\theta$ is the time elapsed since that moment.

It is possible to partition $\Sigma$ into regions $P_{1}, P_{2}, \ldots, P_{n}$ with the property that the symbolic dynamics histories $\boldsymbol{\sigma}=\left\{\sigma_{i}\right\}_{i=-\infty}^{\infty}$ on the sets $P_{\sigma}, \sigma=1, \ldots, n$, has a Markov property, in the sense that

(1) there is a suitable matrix $M_{\sigma, \sigma^{\prime}}$ with entries 0 or 1 , such that if $M_{\sigma_{i}, \sigma_{i+1}} \equiv 1$ for all $i$ then there is a unique point $x$ such that $S^{i} x \in P_{\sigma_{i}}$ : the point $x$ is said to be "coded" by the sequence $\sigma$. And

(2) calling compatible a sequence $\boldsymbol{\sigma}$ with $M_{\sigma_{i}, \sigma_{i+1}} \equiv 1$ then for all points $x$ there is at least one compatible sequence $\boldsymbol{\sigma}$ which codes $x$ and for all but a set of zero volume relative to $\Sigma$ the sequence $\boldsymbol{\sigma}$ is unique (i.e. much as it is the case in the binary representation of real numbers).

The partition $P_{1}, P_{2}, \ldots, P_{n}$ is then called a Markov partition: since the set of exceptions in the correspondence $x \longleftrightarrow \boldsymbol{\sigma}$ has zero volume, the volume distribution can be represented as a probability distribution $\mu_{0}$ over the space of compatible sequences. And the statistics of the evolution of data $\xi$ chosen at random with respect to the distribution $\mu_{0}$, which is the main object of interest, will therefore be represented also by a $S$-invariant probability distribution on the space $\Omega$ of the compatible sequences $\boldsymbol{\sigma},[23]$.

The sets $P_{1}, P_{2}, \ldots, P_{n}$ can be used to represent conveniently the microscopic states of the system: given a precison $h>0$ it is possible to find $N_{h}$ such that the sets

$$
P_{\sigma_{-N_{h}}, \ldots, \sigma_{N_{h}}}=\bigcap_{j=-N_{h}}^{N_{h}} S^{-j} P_{\sigma_{j}}
$$

have a diameter $<h$. Therefore the (nonempty) sets $\Delta=P_{\sigma_{-N h}, \ldots, \sigma_{N_{h}}}$ can be conveniently used as "cells" to describe the evolution, when the size $h$ is small enough for considering acceptable to neglect the variations of the (few) interesting observables within the $\Delta$ 's.

The evolution $S$ will stretch $\Delta$ along the unstable planes and compress it along the stable ones: it will map $\Delta=P_{\sigma_{-N_{h}}, \ldots, \sigma_{N_{h}}}$ inside the union of the $n$ sets $\cup_{\sigma} P_{\sigma_{-N_{h}+1}, \ldots, \sigma_{N_{h}}, \sigma}$.

We then imagine that the cell $\Delta$ is filled by smaller boxes, that will be called microscopic cells or simply $m i$ crocells, of equal volume, which under the action of $S$ are transformed into boxes contained in only one of the $n$ sets $\Delta_{\sigma}=P_{\sigma_{-N_{h}+1}, \ldots, \sigma_{N_{h}}, \sigma}$. The microcells, which in a simulation could be identified with the integers defining them in the computer memory, should be thought of as arranged in layers adjacent to unstable planes of $S$ and are mapped into microcells of the corresponding layers in the $n$ cells $\Delta_{\sigma}$.

Since the evolution, in the average, contracts phase space the layers will merge under the action of $S$ so that the number of microcells will initially decrease; but eventually in each cell $\Delta$ will survive layers of microcells whose collection will be mapped one to one into itself: the latter collection of microcells is a representation of the attractor within the precision $h$. This is illustrated symbolically in Fig.1.

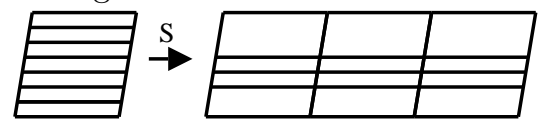

Fig.1: The lines symbolize arrays of microcells $\Delta$ : the ones in the left drawing are stretched and merged by the time evolution into arrays that end up in three new boxes.

For consistency the number of microcells that is eventually found in each cell $\Delta$ is inversely proportional to the expansion rate $\Lambda_{e}(\Delta)^{-1}$ of the surface elements on the unstable manifold in $\Delta$ : it will be denoted $\mathcal{N} \Lambda_{e}(\Delta)^{-1}$

The time evolution $S$ can then be represented as a permutation of the $\mathcal{N} \sum_{\Delta} \Lambda_{e}(\Delta)^{-1}$ microcells on the attractor:

(1) give a rule to select the $\mathcal{N} \Lambda_{e}(\Delta)^{-1}$ microcells in $\Delta=P_{\sigma_{-N_{h}}, \ldots, \sigma_{N_{h}}}$ and to partition them into $n$ groups labeled by $\sigma=1, \ldots, n$, each containing a fraction $\pi_{\sigma}=\frac{\Lambda_{e}^{-1}\left(\Delta_{\sigma}\right)}{\sum_{\sigma^{\prime}} \Lambda_{e}^{-1}\left(\Delta_{\sigma^{\prime}}\right)}$ of the microcells selected in $\Delta$ and 
(2) establish a correspondence $\bar{S}$ between the microcells in the group labeled $\sigma$ and a subset the ones in $\Delta_{\sigma}=P_{\sigma_{-N_{h}+1}, \ldots, \sigma_{N_{h}}, \sigma}$

(3) approximate $S$ by replacing it by $\bar{S}$.

Certainly there is a lot of ambiguity in deciding how to set up the selection and the correspondence: but for the purposes of a description of dynamics with precision $h$ the ambiguity has no consequence. Note that in simulations the microcells selection is implicitly prescribed by the program, and certainly changes quite substantially by any small change of the program or by a change of the computer used. By definition of program the evolution $S$ is replaced by a map of microcells (in huge number even in simple simulations): the map is not invertible but being a map of a finite set into itself it is eventually reduced to a permutation of a subset of the microcells.

Transitivity of the compatibility matrix $M$ implies that the permutation of the microcells on the attractor can be chosen cyclic: therefore the stationary distribution $\mu$ will be approximated by the uniform distribution on the attractor: this is a picture which seems close to BoLtzMANN's conception of discretness and extends the ideas behind the ergodic hypothesis to more general dynamical systems, [11, 24].

\section{A BIT OF HISTORY.}

The discovery of the probability distribution $\mu$ that describes the statistics of the stationary states of dynamical systems with confined evolution did not follow the path discussed in Sec.4: of course every theorem is preceded by a heuristic intuition and the exact genesis of the ideas should be asked to their Authors at least to the ones present here. But there is no certainty that they will give a faithful account, as it is well known that recollection of past events in the human mind, even important ones, tends to be modified as years pass and new events occur.

A possible history about BolTzManN's ergodic hypothesis and theory of ensembles is presented in Sec.1,2.

The theory of hyperbolic transitive systems is much more recent: they were formally introduced by ANOSOV who proved the stability of the notion under perturbations: a hyperbolic transitive system remains such if slightly perturbed.

The existence of a well defined statistics for almost all initial data was heralded, [25], by the work of ADLER and WeISS on the area preserving map of the torus $S\left(\varphi_{1}, \varphi_{2}\right)=\left(\varphi_{1}+\varphi_{2}, \varphi_{1}+2 \varphi_{2}\right)$ : they define and make use of a Markov partition. The idea was independently developed by SinAI, [23, 26], who treated the general case of an Anosov map, building Markov partitions and using them to prove the existence of a privileged distribution $\mu$ giving the statistics of all initial data but a set of zero volume.
A remarkable property of the distribution $\mu$ emerges when it is regarded as a probability distribution on the compatible sequences $\boldsymbol{\sigma}$ which code the points $\mathbf{x}$ phase space. Namely it is a "Gibbs distribution", in the sense of probability theory, with a short range potential: this is, essentially, a Markov process with finitely many states, i.e. an object that is very well understood, [27]. The surprising consequence is that Anosov systems are "completely integrable" in the sense that we can compute essentially everything at least in principle, [23, 28]. They become a paradigm for chaotic evolutions in the same sense in which harmonic oscillators are a paradigm for ordered motions.

Hyperbolicity is a strong property which in practice is not satisfied in physical systems. Therefore Bowen, [29], and RuelLe, [30, 31], developed a theory for systems that are hyperbolic in a much weaker sense: these are systems with axiom $A$ attractors. Also for such systems it is possible to define a natural distribution that describes the statistics of all but a set of zero volume of initial data and the distribution can be studied by an extension of SinAI's methods.

The natural distribution has since been called the $S R B$ distribution and RUELLE has proposed, in the very similar context of turbulence theory, and at least three years earlier than it appeared in print $[31,32]$, that in general there should be a unique distribution (or possibly a finite number of them) describing the experimental statistics of motions: it should be the distribution giving the asymptotic behavior of motions with arbitrary initial data apart from a set of zero volume.

\section{DEVELOPMENTS.}

More recently CoHen and myself, [33] and see also [11], proposed cutting a "Gordian node" by an hypothesis which extends the viewpoint expressed by RuELLE in [34] "... while one would be very happy to prove ergodicity because it would justify the use of Gibbs' microcanonical ensemble, real systems perhaps are not ergodic but behave nevertheless in much the same way and are well described by Gibbs' ensemble...":

Chaotic hypothesis: The asymptotic motions of a confined chaotic mechanical system develop on an attracting set on which motion can be considered a mixing Anosov flow.

Of course this applies in particular to Hamiltonian systems (where the attracting set is the full energy surface) and it implies ergodicity: hence the whole body of equilibrium Statistical Mechanics; furthermore it puts on the same level equilibrium and non equilibrium.

This interpretation of RuELLE's ideas, [32, 34], has some applications because it implies a formal expression of the average values of the observables. Even though the expression is not (yet ?) computable in any interesting 
case it may be useful to establish relations between average values. For instance implications of a microscopic symmetry on macroscopic observables might be found from the formal (even if not practically computable) expression of the SRB distribution.

An example is obtained by considering an Anosov system $(\mathcal{F}, S)$, with $\mathcal{F}$ a smooth bounded manifold and $S$ a smooth transitive hyperbolic map of $\mathcal{F}$. Let

$$
\sigma(x)=-\log \left|\operatorname{det} \partial_{x} S(x)\right|
$$

be the phase space contraction rate and let $\mu$ be the SRB distribution; suppose

(a) dissipativity, i.e. $\sigma_{+}=\int \sigma(y) \mu(d y)>0$, and

(b) time reversal symmetry in the sense that there is a smooth isometry $I$ such that $I S=S^{-1} I$.

Define

$$
p=\frac{1}{\tau} \sum_{j=0}^{\tau-1} \frac{\sigma\left(S^{j} x\right)}{\sigma_{+}}
$$

then the following theorem holds, [33],

Fluctuation theorem: With respect to the SRB distribution the observable $p$ satisfies a large deviation property (see below) with a rate function $\zeta(p)$ which is analytic and convex in an interval $\left(-p^{*}, p^{*}\right)$, for a suitable $p^{*} \geq 1$, where it exhibits the symmetry property

$$
\zeta(-p)=\zeta(p)-p \sigma_{+}
$$

This means that the probability, with respect to the SRB distribution $\mu$ of $(\mathcal{F}, S)$, that $p$ is inside an interval $[a, b] \subset\left(-p^{*}, p^{*}\right)$ is $P_{a, b}$ with $\lim _{\tau \rightarrow \infty} \frac{1}{\tau} \log P_{a, b}=$ $\max _{p \in[a, b]} \zeta(p)$.

Existence and analyticity of $\zeta(p)$ is part of the quoted general results of SinAI, while the symmetry 11 was pointed out in [33] in an attempt to explain the numerical results of an earlier computer experiment [35]. The interest of the theorem lies in the fact that it is a symmetry property: hence it holds without any free parameter.

The theorem can be extended to mixing Anosov flows, [36], and therefore, via the chaotic hypothesis and if $\sigma(x)$ is the phase space contraction rate defined in 5 , it becomes a property of essentially any system which is chaotic, dissipative and reversible.

\section{ENTROPY?}

Interest in the properties of the observable $\sigma(x), 5$ for flows and 10 for maps, arose in several molecular dynamics simulations in which it was naturally related to the entropy creation rate.
A natural question is whether a definition of entropy can be extended to nonequilibrium stationary states in analogy with the corresponding definition for equilibrium states (which are a very special case of stationary states).

The identification between the SRB distributions and distributions giving equal probability to the microcells in the attractor shows that it should be possible, at least, to define a function which is a Lyapunov function for the approach to stationarity: this would be an extension of the $H$-theorem of Boltzmann. However equality between the $H$ function evaluated in equilibrium states and thermodynamic entropy might be a coincidence, important but not extendible to non equilibrium (hence not necessary). Arguments in this direction can be found in the literature, $[37,38]$, and here the controversial aspects of this matter will not be touched, [39].

It will be worth however to enter into more details about why $\sigma(x)$ has been called entropy creation rate. This is simply because in several experiments it had such an interpretation, being the ratio between a quantity that could be identified with the work per unit time done by the noncoservative forces stirring the system divided by a quantity identified with temperature of the thermostat providing the forces that extract the energy input from the stirring forces. The experiments were simulations and from many sides critiques were expressed because the interpretation seemed closely tied to the explicit form of the thermostats models, often considered "unphysical".

Furthermore the explicit dependence on the equations of motion makes the identification of $\sigma(x)$ with the entropy creation rate quite useless if the aim is to compare the theory with experiments different from simulations because in real experiments (i.e. on experiments on matter distinct from impressive arrays of transistors) there usually is no explicit model of thermostat force and it is difficult to evaluate $\sigma(x)$. And it might turn out that the identification of $\sigma(x)$ with entropy creation rate is closely related to the special models considered.

A simple, but quite general, model of thermostatted system may be useful to show that, while we should expect that there is a relation between entropy creation rate and phase space contraction, still the two notions are quite different.

The system consists in $N \equiv N_{0}$ particles in a container $\mathcal{C}_{0}$ and of $N_{a}$ particles in $n$ containers $\mathcal{C}_{a}$ which play the role of thermostats: their positions will be denoted $\mathbf{X}_{a}, a=0,1, \ldots, n$, and $\mathbf{X} \stackrel{\text { def }}{=}\left(\mathbf{X}_{0}, \mathbf{X}_{1}, \ldots, \mathbf{X}_{n}\right)$. Interactions will be described by a potential energy

$$
W(\mathbf{X})=\sum_{a=0}^{n} U_{a}\left(\mathbf{X}_{a}\right)+\sum_{a=1}^{n} W_{a}\left(\mathbf{X}_{0}, \mathbf{X}_{a}\right)
$$

i.e. thermostats particles only interact indirectly, via the system. All masses will be $m=1$, for simplicity.

The particles in $\mathcal{C}_{0}$ will also be subject to external, possibly nonconservative, forces $\mathbf{F}\left(\mathbf{X}_{0}, \boldsymbol{\Phi}\right)$ depending on a few strength parameters $\boldsymbol{\Phi}=\left(E_{1}, E_{2}, \ldots\right)$. It is convenient to imagine that the force due to the confining 
potential determining the region $\mathcal{C}_{0}$ is included in $\mathbf{F}$, so that one of the parameters is the volume $V=\left|\mathcal{C}_{0}\right|$. See Fig.2 below.

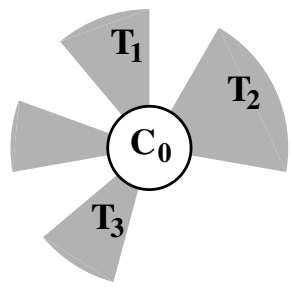

Fig. 2 The reservoirs occupy finite regions outside $C_{0}$, e.g. sectors $C_{a} \subset R^{3}, a=1,2 \ldots$ Their particles are constrained to have a total kinetic energy $K_{a}$ constant, by suitable forces $\boldsymbol{\vartheta}_{a}$, so that the reservoirs "temperatures" $T_{a}$, see 14 , are well defined.

The equations of motion will be, assuming the mass $m=1$,

$$
\begin{aligned}
\ddot{\mathbf{X}}_{0 i} & =-\partial_{i} U_{0}\left(\mathbf{X}_{0}\right)-\sum_{a} \partial_{i} U_{a}\left(\mathbf{X}_{0}, \mathbf{X}_{i}\right)+\mathbf{F}_{i} \\
\ddot{\mathbf{X}}_{a i} & =-\partial_{i} U_{a}\left(\mathbf{X}_{a}\right)-\partial_{i} U_{a}\left(\mathbf{X}_{0}, \mathbf{X}_{i}\right)-\alpha_{a} \dot{\mathbf{X}}_{a}
\end{aligned}
$$

where the last force term $-\alpha_{a} \dot{\mathbf{X}}_{a}$ is a phenomenological force that implies that the thermostats particles keep constant kinetic energies:

$$
K_{a}=\sum_{j=1}^{N_{a}} \frac{1}{2}\left(\dot{\mathbf{X}}_{j}^{a}\right)^{2} \stackrel{\text { def }}{=} \frac{3}{2} N_{a} k_{B} T_{a} \stackrel{\text { def }}{=} \frac{3}{2} N_{a} \beta_{a}^{-1}
$$

where the parameters $T_{a}$ should define the thermostats temperatures and $\alpha_{a}$ can, for instance, be defined by

$$
-\alpha_{a} \stackrel{\text { def }}{=} \frac{L_{a}-\dot{U}_{a}}{3 N_{a} k_{B} T_{a}}
$$

where $L_{a}=-\partial \mathbf{X}_{a} W_{a}\left(\mathbf{X}_{0}, \mathbf{X}_{a}\right) \cdot \dot{\mathbf{X}}_{a}$ is the work done per unit time by the forces that the particles in $\mathcal{C}_{0}$ exert on the particles in $\mathcal{C}_{a}$.

The exact form of the forces that have to be added in order to insure the kinetic energies constancy should not really matter, within wide limits. But this is a property that is not obvious and which is much debated. The above thermostatting forces choice is dictated by Gauss' least effort principle for the constraints $K_{a}=$ const, see appendix 9.4 in [11]: this is a criterion that has been adopted in several simulations, [40]. Independently of Gauss' principle it is immediate to check that if $\alpha_{a}$ is defined by 15 then the kinetic energies $K_{a}$ are strictly constants of motion.

The work $L_{a}$ in 15 will be interpreted as heat $\dot{Q}_{a}$ ceded, per unit time, by the particles in $\mathcal{C}_{0}$ to the $a$-th thermostat (because the "temperature" of $\mathcal{C}_{a}$ remains constant, hence the thermostats can be regarded in thermal equilibrium). The entropy creation rate due to heat exchanges between the system and the thermostats can, therefore, be naturally defined as

$$
\sigma^{0}(\dot{\mathbf{X}}, \mathbf{X}) \stackrel{\text { def }}{=} \sum_{a=1}^{N_{a}} \frac{\dot{Q}_{a}}{k_{B} T_{a}}
$$

It should be stressed that here no entropy notion is introduced for the stationary state: only variation of the thermostats entropy is considered and it should not be regarded as a new quantity because the thermostats should be considered in equilibrium at a fixed temperature.

The question is whether there is any relation between $\sigma_{0}$ and the phase space contraction $\sigma$ of 5 . The latter can be immediately computed and is (neglecting $\left.O\left(\min _{a>0} N_{a}^{-1}\right)\right)$

$$
\sigma^{\Gamma}(\dot{\mathbf{X}}, \mathbf{X})=\sum_{a>0} \frac{3 N_{a}-1}{3 N_{a}} \frac{\dot{Q}_{a}-\dot{U}_{a}}{k_{B} T_{a}}=\sum_{a>0} \frac{\dot{Q}_{a}}{k_{B} T_{a}}-\dot{U}(17)
$$

where $U=\sum_{a>0} \frac{3 N_{a}-1}{3 N_{a}} \frac{U_{a}}{k_{B} T_{a}}$. Hence in this example in which the thermostats are "external" to the system volume (unlike to what happens in the common examples in which they act inside the volume of the system), the phase space contraction is not the entropy creation rate, [41]. However it differs from the entropy creation rate by a total derivative.

The latter remark implies that if the chaotic hypothesis is accepted for the system in Fig.2 then, assuming $U_{a}$ bounded (for simplicity, see [41, 42] for more general cases) it is $\sigma_{+}=\left\langle\sigma_{0}\right\rangle$ because the derivative $\dot{U}$ contributes $\frac{1}{\tau}(U(\tau)-U(0)) \underset{\tau \rightarrow \infty}{\longrightarrow} 0$ and also the observable $p$, in the continuous time extension of 10 , [36], has the same rate function as the observable $p=$ $\frac{1}{\tau} \int_{0}^{\tau} \sigma_{0}\left(S_{t} x\right) d t \equiv \frac{1}{\tau} \int_{0}^{\tau} \sigma\left(S_{t} x\right) d t+O\left(\tau^{-1}\right)$. Since the equations of motion 13 are time reversible (a rather general property of Gaussian constraints, with $I$ being here simply velocity reversal) it follows that the "physical entropy creation" 16 has a fluctuations rate $\zeta(p)$ satisfying the fluctuation relation 11 .

This is relevant because the definition 16 has meaning independently of the equations of motions and can therefore be suitable for experimental tests. [41, 43]. The above is just a model of thermostats: other interesting models have been proposed based on purely Hamiltonian interactions at the price of relying on thermostats of infinite size, see [44-46].

\section{EXTENSIONS OF BOLTZMANN'S $H$-THEOREM}

The above analysis does not require a notion of entropy to be defined for stationary states.

There is, however, another key contribution of Boltzmann to Statistical Mechanics, briefly mentioned above. This is the Boltzmann's equation and the relative $H$ theorem, [47]. 
The theorem has attracted deep interest because of its philosophical implications. For our purposes it is important because it provides a theory of approach to equilibrium and therefore it is one of the first results on nonequilibrium.

It is useful to stress that the definition of $H$ is given in the context of the approach to equilibrium and BoLTZMANN never applied it (nor, perhaps, meant to apply it) to the approach to other stationary states and to their theory. The equality of the value of $H$ with the thermodynamic entropy when evaluated on the equilibrium state raised the hope that it could be possible to define entropy for systems out of equilibrium and even if not in stationary state. The idea emerged clearly already from the foundational papers on the Boltzmann equation (" $\log P$ was well defined whether or not the system is in equilibrium, so that it could serve as a suitable generalization of entropy", p. 82 in [48] and p. 218 in [21]) and many attempts can be found in the literature to define entropy for systems out of equilibrium in stationary states or even in macroscopically evolving states.

Strictly speaking the implication that can be drawn from the works on the Boltzmann's equation is that a rarefied gas started in a given configuration evolves in time so that the average values of the observables, at least of the few of interest, acquire an asymptotic value which is the same as the one that can be computed from a probability distribution maximizing a function $H$.

The acquisition of an asymptotic value by the averages of the observables is a property expected to hold also when the asymptotic state is a nonequilibrium stationary state. And it is natural to think that also in such cases there will be a function that approaches monotonically an asymptotic value signaling that the few observables of interest approach their asynptotic average.

As remarked above the SRB distribution is a uniform distribution over the attractor: therefore it verifies a variational property and this can be used to define a Lyapunov function that evolves towards a maximum, [37]. Let $\boldsymbol{\eta}=\left(\sigma_{-N_{h}}, \ldots, \sigma_{N_{h}}\right)$ and $H \stackrel{\text { def }}{=} \frac{1}{\tau} \sum_{\boldsymbol{\eta}}-p_{\boldsymbol{\eta}}\left(\log p_{\boldsymbol{\eta}}+\right.$ $\left.\log \Lambda_{\tau}\left(\boldsymbol{\eta}^{-1}\right)\right)$ where $p_{\boldsymbol{\eta}}$ denotes the fraction of microcells that can be found in the cell $\Delta=P_{\boldsymbol{\eta}}=\cap_{k=-N_{h}}^{N_{h}} S^{-k} P_{\eta_{k}}$ after a time of $\tau$ units has elapsed starting from an initial distribution $p_{\boldsymbol{\eta}}^{0}$ (typically a uniform distribution over the microcells in a single cell $\Delta^{0}$ ). This is a quantity that tends to a maximum as time evolves (reaching it when the $p_{\boldsymbol{\eta}}$ have the value of the SRB distribution and the maximum equals, therefore, the logarithm of the number of microcells on the attractor).

Therefore the quantity $H$ tends in the average to a maximum and it can be regarded as an instance of an $H$-function. However the maximum depends on the precision $h$ of the coarse graining defined by the partition of phase space by the cells $\Delta$. Changing the precison several changes occur which have to be examined if a meaning other than that of a Lyapunov function has to be given to $H$. The analysis in [37] points out that $H$ changes with the precision $h$ in a trivial way (i.e. by an additive constant, independent of the control parameters of the system and depending only on the precision $h$ ) if the SRB state on which it is evaluated is an equilibrium state. In the latter case it is proportional to the logarithm of the phase space volume that can be visited. In the nonequilibrium cases however $H$ changes when the precision $h$ changes by additive quantities that are not just functions of $h$ but depend on thermodynamic quantities, (like average energy, temperatures, etc. .), [37].

This indicates that while not excluding the possibility of existence of Lyapunov functions, see [49], indicating the approach to equilibrium (within a given precision $h$ ) the identity of the $H$ function with entropy, i.e. its identity with a function of the state parameters of the system, is possible only when the state is in an equilibrium state. My interpretation of this analysis, based once more on a discrete point of view on the problem, is that one should not insist in looking for a notion of entropy in systems out of equilibrium, [37].

If so once again BolTzMAnN's attitude to consider phase space as discrete and in general to deny reality to the continua might have led to insights into difficult questions.

\section{CONCLUSION}

BoltzmanN's contribution to the theory of ensembles and to the mechanical interpretation of heat and Thermodynamics was based on a discrete conception of the continuum: his staunch coherence on this view has been an essential aspect of the originality of his thought.

It is in fact a method of investigation which is still very fruitful and used in various forms when "cut-offs" or "regularizations" are employed in the most diverse fields. In my view it has been and still is important in the recent developments in the theory of nonequilibrium stationary states. The Fluctuation theorem and its various interpretations, extensions and applications (to Onsager reciprocity at non zero forcing, to Green-Kubo formulae, to fluid Mechanics, Turbulence and Intermittency, see $[11,23,50])$ is, hopefully, only an example.

It is interesting in this context recall a few quotes from BoltzmanN

"Through the symbols manipulations of integral calculus, which have become common practice, one can temporarily forget the need to start from a finite number of elements, that is at the basis of the creation of the concept, but one cannot avoid it";

see p. 227 in [51], or in the same page:

"Differential equations require, just as atomism does, an initial idea of a large finite number of numerical values and points ...... Only afterwards it is maintained that the picture never represents phenomena exactly but merely approximates them more and more the greater the 
number of these points and the smaller the distance between them. Yet here again it seems to me that so far we cannot exclude the possibility that for a certain very large number of points the picture will best represent phenomena and that for greater numbers it will become again less accurate, so that atoms do exist in large but finite number."

and, see p. 55 in [51]:

"This naturally does not exclude that, after we got used once and for all to the abstraction of the volume elements and of the other symbols [of Calculus] and once one has studied the way to operate with them, it could look handy and luring, in deriving certain formulae that Volkmann calls formulae for the coarse phenomena, to forget completely the atomistic significance of such abstractions. They provide a general model for all cases in which one can think to deal with $10^{10}$ or $10^{10^{10}}$ elements in a cubic millimeter or even with billions of times more; hence they are particularly invaluable in the frame of Geometry, which must equally well adapt to deal with the most diverse physical cases in which the number of the elements can be widely different. Often in the use of all such models, created in this way, it is necessary to put aside the basic concept, from which they have overgrown, and perhaps to forget it entirely, at least temporarily. But I think that it would be a mistake to think that one could become free of it entirely."

And the principle was really applied not only in the conception of the ergodic hypothesis, $[2,6]$, but also in the deduction of the Boltzmann's equation which BoLTzMANN felt would be clarified by following discretization methods (in energy) inspired by those employed in the "elegant solution of the problem of string-vibrations" of LAgRAnge, or in Stefan's study of diffusion or in RIEMANN's theory of mean curvature, [47] and in various discussions of the heat theorem, [21].

The above conception of the infinitesimal quantities, rooted in the early days of Calculus when " $d x$ " was regarded as infinitely small and yet still of finite size (in apparent, familiar, logical contradiction), is an important legacy that should not be forgotten in spite of the social pressure that induces all of us to identify clarity of physical understanding with continuous models of reality.

\section{Appendix: Temperature and kinetic energy, [3, 15]}

The first attempts at a kinetic explanation of the properties of gases came following the experiments by BOYLE, (1660), on the gas compression laws. The laws established that "air" had elastic properties and that there was inverse proportionality between pressure and volume: a theory that was considered also by NewTON. It was D. Bernoulli, (1720), who abandoned the view, espoused by NEWTON, that the atoms were arranged on a kind of lattice repelling each other (with a force inversely proportional to their distances to agree with Boyle's law, but extending only to the nearest neighbors). BERNOULLI imagined the atoms to be free and that pressure was due to the collisions with the walls and proportional to the square of the average speed proposing that a correct definition of temperature should be based on this property.

In 1816 AVOGADRO established that, for rarefied gases, the ratio $p V / T$ is proportional to the number of atoms or molecules via a universal constant. This was a striking result, explaining the anomalies in the earlier theory of DALTON and allowing, besides the definition of the Avogadro's number, the correct determination of the relative molecular and atomic weights. It openend the way to the definition of absolute temperature, independently of the special gas-thermometer employed, and to the principle of energy equipartition and to the later works of Waterston, Clausius, Boltzmann, among others.

The attempt of LAPlACE, (1821), proposed an elaborate scheme in which the atoms, still essentially fixed in space at average distance $r$ would contain a quantity $c$ of caloric and would interact with a short range force proportional to the product of their quantity of caloric and depending on the distance. Identifying the caloric $c$ with the a fixed amount contained in each atom would have led to a gas law with $p$ proportional to the square of the density $\rho$, i.e. to $\rho^{2} c^{2}$; but this was avoided by supposing that the amount of caloric $c$ in each molecule was determined by an equilibrium between the amount of caloric emitted by a molecule and the caloric received by it (emitted from the other molecules) which was supposed to depend only on the temperature, see [3].

The theory of LAPLACE did not sound convincing and the work of BERNOULLI went unnoticed; the same was the fate of the work of Herapath, (1820), who again proposed, without knowing BERNOULLI's theory, that the atoms were free and pressure was due to collisions with the walls; however he assumed that pressure was proportional to the average momentum rather than kinetic energy obtaining an incorrect definition of absolute temperature. In any event his work was rejected by the Philosophical transactions of the Royal Society of London and published on the Annals of Philosophy falling into oblivion for a while.

In 1845 WATERSTONE, unaware of both BeRnoulli and Herapath but (likely) familiar with Avogadro's work, proposed the theory of gases with the correct identification of pressure as proportional to the average kinetic energy and the density, introducing also a rather detailed conception of te interatomic forces taking up ideas inspired by Mossotti (who probably had also made AvOGADRO and Italian science better known in England during his political exile). Unfortunately he submitted it to the Philosophical transactions of the Royal Society which readily rejected it and remained unpublished, until it was rediscovered much later (1892, by RAILEIGH).

In the 1840's, through the work of MEYER, Joule, HELMOLTZ and others the energy conservation principle was established with the consequent identification of heat as a form of energy convertible into mechanical work forcing (reasonable) physicists to abandon the hypothesis of the existence of caloric as a conserved entity. 
The theory of gases begun to be really accepted with the work of KRÖNIG, (1856), who clearly proposed identifying temperature with average kinetic energy of molecules. His work became well known as it appeared to have prompted the publication of CLAUSIUS's paper of (1857), who had independently reached the same conclusions and gone much further. Not only Clausius went quite far in establishing energy equipartition (completed by MAXWELL in 1860) but he introduced a basic concept of kinetic theory: the mean free path. Thus making clear the role of collisions in gas theory: they lead to prediction and to a first understanding of the phenomenon of diffusion, explaining the apparent paradoxes linked to the earlier assumptions that in rarefied gases collisions could be simply neglected, and also initiate the theory of the transport coefficients.

The latter papers, one century after the too far in advance (over his time) work of BERNOULLI, gave origin to kinetic theory in the sense we intend it still now, and stimulated also the related investigations of MAXwELL.
Therefore Maxwell (1859) and a little later BoltzMANN (1866) could start their work taking for granted the well established identity between temperature and average kinetic energy for gases extending it to hold in all systems in equilibrium (rarefied or not). This key view was not destined to have a long life: the advent of Quantum Mechanics would prove that proportionality between average kinetic energy and temperature could only be approximate and to hold if quantum corrections to Atomic Mechanics were negligible, see [11]. Nevertheless the identification of temperature and kinetic energy plaid (and still plays, whenever quantum effects are negligible) an essential role not only in classical Statistical Mechanics but also in the discovery of Quantum Mechanics, which was heralded by the failure of the related equipartition of energy.

Source of the talk at the BoltzMANN's LEGACY international symposium at ESI, Vienna, 7-9 June, 2006
[1] L. Boltzmann, Über die mechanische Bedeutung des zweiten Haupsatzes der Wärmetheorie, vol. 1 of Wissenschaftliche Abhandlungen, ed. F. Hasenöhrl (Chelsea, New York, 1968).

[2] L. Boltzmann, Über das Wärmegleichgewicht zwischen mehratomigen Gasmolekülen, vol. 1 of Wissenschaftliche Abhandlungen, ed. F. Hasenöhrl (Chelsea, New York, 1968).

[3] S. Brush, History of modern physical sciences: The kinetic theory of gases (Imperial College Press, London, 2003).

[4] L. Boltzmann, Studien über das Gleichgewicht der lebendigen Kraft zwischen bewegten materiellen Punkten, vol. 1 of Wissenschaftliche Abhandlungen, ed. F. Hasenöhrl (Chelsea, New York, 1968).

[5] L. Boltzmann, Entropie und Wahrscheinlichkeit, vol. 286 of Ostwalds Klassiker der Exacten Wissenschaften, Ed. D. Flamm (Verlag Harri Deutsch, ISBN 3-8171-3286-7, Frankfurt am Main, 2000).

[6] L. Boltzmann, Einige allgemeine sätze über Wärmegleichgewicht, vol. 1 of Wissenschaftliche Abhandlungen, ed. F. Hasenöhrl (Chelsea, New York, 1968).

[7] L. Boltzmann, Bemerkungen über einige Probleme der mechanischen Wärmetheorie, vol. 2 of Wissenschaftliche Abhandlungen, ed. F. Hasenöhrl (Chelsea, New York, 1968).

[8] H. Helmholtz, Prinzipien der Statistik monocyklischer Systeme, vol. III of Wissenschaftliche Abhandlungen (Barth, Leipzig, 1895).

[9] H. Helmholtz, Studien zur Statistik monocyklischer Systeme, vol. III of Wissenschaftliche Abhandlungen (Barth, Leipzig, 1895).

[10] L. Boltzmann, Über die Eigenshaften monozyklischer und anderer damit verwandter Systeme, vol. 3 of Wissenschaftliche Abhandlungen (Chelsea, New-York, 1968).

[11] G. Gallavotti, Statistical Mechanics. A short treatise (Springer Verlag, Berlin, 2000).
[12] G. Gallavotti, Journal of Statistical Physics 78, 1571 (1995).

[13] M. Fisher, Archive for Rational Mechanics and Analysis 17, 377 (1964).

[14] D. Ruelle, Communications in Mathematical Physics 9, 267 (1968).

[15] S. Brush, The kind of motion that we call heat, (I, II) (North Holland, Amsterdam, 1976).

[16] P. Ehrenfest and T. Ehrenfest, The conceptual foundations of the statistical approach in Mechanics (Dover, New York, 1990).

[17] L. Boltzmann, Reply to Zermelo's Remarks on the theory of heat, vol. 1 of History of modern physical sciences: The kinetic theory of gases, ed. S. Brush (Imperial College Press, London, 2003).

[18] L. Boltzmann, Lectures on gas theory, English edition annotated by S. Brush (University of California Press, Berkeley, 1964).

[19] W. Thomson, Proceedings of the Royal Society of Edinburgh 8, 325 (1874).

[20] D. Levesque and L. Verlet, Journal of Statistical Physics 72, 519 (1993).

[21] L. Boltzmann, Über die Beziehung zwischen dem zweiten Hauptsatze der mechanischen Wärmetheorie und der Wahrscheinlichkeitsrechnung, respektive den Sätzen über das Wärmegleichgewicht, vol. 2 of Wissenschaftliche Abhandlungen, ed. F. Hasenöhrl (Chelsea, New York, 1968).

[22] D. Ruelle, Journal of Statistical Physics 85, 1 (1996).

[23] G. Gallavotti, F. Bonetto, and G. Gentile, Aspects of the ergodic, qualitative and statistical theory of motion (Springer Verlag, Berlin, 2004).

[24] G. Gallavotti, Journal of Statistical Physics 84, 899 (1996).

[25] R. Adler and B. Weiss, Similarity of automorphism of the tours, vol. 98 of Memoirs of the American Mathematical Society (American Mathematical Society, 1970).

[26] Y. Sinai, Functional Analysis and Applications 2, 64 
(1968).

[27] Y. Sinai, Functional analysis and Applications 2, 70 (1968).

[28] Y. Sinai, Russian Mathematical Surveys 27, 21 (1972).

[29] R. Bowen, American Journal of Mathematics 92, 725 (1970).

[30] R. Bowen and D. Ruelle, Inventiones Mathematicae 29, 181 (1975).

[31] D. Ruelle, American Journal of Mathematics 98, 619 (1976).

[32] D. Ruelle, Annals of the New York Academy of Sciences 357, 1 (1980).

[33] G. Gallavotti and E. Cohen, Physical Review Letters 74, 2694 (1995).

[34] D. Ruelle, Ergodic theory, vol. Suppl X of The Boltzmann equation, ed. E.G.D Cohen, W. Thirring, Acta Physica Austriaca (Springer, New York, 1973).

[35] D. Evans, E. Cohen, and G. Morriss, Physical Review Letters 70, 2401 (1993).

[36] G. Gentile, Forum Mathematicum 10, 89 (1998).

[37] G. Gallavotti, Chaos 14, 680 (2004).

[38] G. Gallavotti, Communication in Mathematical Physics 224, 107 (2001).

[39] S. Goldstein and J. Lebowitz, Physica D 193, 53 (2004).

[40] D. Evans and G. Morriss, Statistical Mechanics of Nonequilibrium Fluids (Academic Press, New-York, 1990).

[41] G. Gallavotti, Chaos 16, $023130(+7)$ (2006).

[42] F. Bonetto, G. Gallavotti, A. Giuliani, and F. Zamponi, Journal of Statistical Mechanics 123, 39 (2006).

[43] F. Bonetto, G. Gallavotti, A. Giuliani, and F. Zamponi, Journal of Statistical Mechanics (cond-mat/0601683) p. P05009 (2006).

[44] C. Jarzynski, Journal of Statistical Physics 98, 77 (1999).

[45] J.P.Eckmann, C. Pillet, and L. R. Bellet, Communications in Mathematical Physics 201, 657 (1999).

[46] D. Ruelle, Communications in Mathematical Physics ??, ?? (2006).

[47] L. Boltzmann, Weitere Studien über das Wärmegleichgewicht unter Gasmolekülen, vol. 1 of Wissenschaftliche Abhandlungen, ed. F. Hasenöhrl (Chelsea, New York, 1968).

[48] M. Klein, The development of Boltzmann statistical ideas, vol. Suppl X of The Boltzmann equation, ed. E.G.D Cohen, W. Thirring, Acta Physica Austriaca (Springer, New York, 1973).

[49] P. L. Garrido, S. Goldstein, and J. L. Lebowitz, Physical
Review Letters 92, $050602(+4)$ (2005).

[50] G. Gallavotti, cond-mat/0402676 (2004).

[51] L. Boltzmann, Theoretical Physics and philosophical writings, ed. B. Mc Guinness (Reidel, Dordrecht, 1974).

[52] J. Gibbs, Elementary principles in statistical mechanics (reprint) (Schribner, Cambridge (USA), 1902).

[53] [The paper [6] is a key work, albeit admittedly obscure: in modern notations it considers a system of equations of motion with dimension $n$ and 0 divergence admitting $n-k$ constants of motion, $\varphi_{k+1}, \ldots, \varphi_{n}$, and are decribed by coordinates $s_{1}, \ldots, s_{n}$. Then the distribution proportional to $\prod_{j=k+1}^{n} \delta\left(\varphi_{j}-a_{j}\right) \cdot \prod_{i=1}^{n} d s_{i}$ is invariant and can be written $\prod_{i=1}^{k} d s_{i} \cdot \frac{1}{\left|\operatorname{det} \partial\left(\varphi_{n-k+1}, \ldots, \varphi_{n}\right)\right|}$ where the last denominator denotes the Jacobian determinant ("last multiplier") of $\varphi_{n-k+1}, \ldots, \varphi_{k}$ with respect to $s_{k+1}, \ldots, s_{n}$ evaluated at the given values $a_{k+1}, \ldots, a_{n}$ of the constants of motion (BOLTZMANN calls this an instance of the "last multiplier principle" of JACOBI). If the system has only one constant of motion, namely the energy $H=\chi+\psi$ with $\chi=$ potential energy and $\psi=$ kinetic energy, this is the microcanonical distribution, as also recognized by GIBBS in the introduction of his book, [52] (where he quotes [6], but giving to it the title of its first section).]

[54] [Still today a different interpretation of the word "ensemble" is widely used: the above is based on what BoLTZMANN calls "Gattung von Monoden", see p.132, 1. 14 of [10]: unfortunately he is not really consistent in the use of the name "monode" because, for instance in p. 134 of the same reference, he clearly calls "monode" a collection of invariant distributions rather than a single one; further confusion is generated by a typo on p. 132, 1 . 22 , where the word "ergode" is used instead of "holode" while the "ergode" is defined only on p. 134. It seems beyond doubt that "holode" and "ergode" were intended by Boltzmann to be collections $\mathcal{E}$ of invariant distributions (parameterized respectively by $U, V$ or by $\left(k_{B} T\right)^{-1}, V$ in modern notations): Gibbs instead called "ensemble" each single invariant distribution, or at least that is what is often stated. It seems that the original names proposed by BOLTZMANN are more appropriate, but of course we must accept calling "microcanonical ensemble" the ergode and "canonical ensemble" the holode, see [11].]

$\mathrm{REVTEX}_{\mathrm{T}}$ 